\title{
Discrepancy estimates for a class of normal numbers
}

\author{
by
}

Yoshinobu Nakai (Kofu) and Iekata Shiokawa (Yokohama)

To the memory of Gerold Wagner

1. Introduction. Let $r \geq 2$ be a fixed integer and let $\theta=0 . a_{1} a_{2} \ldots$ be the $r$-adic expansion of a real number $\theta$ with $0<\theta<1$. Let $N\left(\theta ; b_{1} \ldots b_{l} ; n\right)$ denote the number of a given block $b_{1} \ldots b_{l} \in\{0,1, \ldots, r-1\}^{l}$ appearing in the first $n$ digits $a_{1} a_{2} \ldots a_{n}$. Then $\theta$ is said to be normal to the base $r$ if, for every fixed $l \geq 1$,

$$
R_{n}(\theta)=R_{n, l}(\theta)=\sup _{b_{1} \ldots b_{l}}\left|\frac{1}{n} N\left(\theta ; b_{1} \ldots b_{l} ; n\right)-\frac{1}{r^{l}}\right|=o(1)
$$

as $n \rightarrow \infty$, where the supremum is taken over all $b_{1} \ldots b_{l} \in\{0,1, \ldots, r-1\}^{l}$. Historical surveys on the study of normal numbers can be found in, e.g., [6].

Let $g(t)$ be a polynomial of $t$ with real coefficients such that $g(t)>0$ for $t>0$. We define a real number

$$
\theta_{r}=\theta_{r}(g)=0 . a_{11} a_{12} \ldots a_{1 k(1)} a_{21} a_{22} \ldots a_{2 k(2)} a_{31} \ldots
$$

to be the infinite $r$-adic fraction obtained from the $r$-adic expansion $[g(n)]=$ $a_{n 1} a_{n 2} \ldots a_{n k(n)}$ of the integral part of $g(n)$, which will be written simply as

$$
\theta_{r}=0 .[g(1)][g(2)][g(3)] \ldots
$$

Let $N\left(g(n) ; b_{1} \ldots b_{l}\right)$ denote the number of a given block $b_{1} \ldots b_{l}$ appearing in the $r$-adic expansion of $[g(n)]$.

If $g(t)$ is a nonconstant polynomial with rational coefficients all of whose values for $t=1,2,3, \ldots$ are positive integers, Davenport and Erdős [3] proved that $R_{n}\left(\theta_{10}(g)\right)=o(1)$, namely, $\theta_{10}(g)$ is normal to the base 10 . They did not give explicit estimates for $R_{n}\left(\theta_{r}(g)\right)$. Schoißengeier [11] showed that $R_{n}\left(\theta_{r}(g)\right)=O\left((\log \log n)^{4+\varepsilon} / \log n\right)$. Later, Schiffer [10] improved it by giving the best possible result $R_{n}\left(\theta_{r}(g)\right)=O(1 / \log n)$. In the case of polynomials with real, but not necessarily rational, coefficients, we proved in [9] that $R_{n}\left(r_{r}(g)\right)=O((\log \log n) / \log n)$, which will be replaced in this paper by $O(1 / \log n)$. 
THEOREM. Let $g(t)$ be any nonconstant polynomial with real coefficients such that $g(t)>0$ for all $t>0$. Then for any block $b_{1} \ldots b_{l} \in\{0,1, \ldots$ $\ldots, r-1\}^{l}$, we have

$$
\sum_{n \leq x} N\left(g(n) ; b_{1} \ldots b_{l}\right)=\frac{1}{r^{l}} x \log _{r} g(x)+O(x)
$$

as $x \rightarrow \infty$, where the implied constant depends possibly on $g, l$, and $r$.

Noting that the number of digits in the $r$-adic expansion of $0 .[g(1)][g(2)] \ldots[g(n)]$ is

$$
n \log _{r} g(n)+O(n) \gg \ll n \log n
$$

with $\log _{r} y=\log y / \log r$, we obtain

Corollary. For any $g(t)$ as in the theorem, we have

$$
R_{n}\left(\theta_{r}(g)\right)=O\left(\frac{1}{\log n}\right)
$$

as $n \rightarrow \infty$. In particular, $\theta_{r}(g)$ is normal to the base $r$.

Remark 1. Let us consider a more general function of the following form:

$$
h(t)=\alpha t^{\beta}+\alpha_{1} t^{\beta_{1}}+\ldots+\alpha_{d} t^{\beta_{d}},
$$

where $\alpha$ 's and $\beta$ 's are real numbers with $\beta>\beta_{1}>\ldots>\beta_{d} \geq 0$. We assume that $h(t)>0$ for $t>0$. If $h(t)$ is not a polynomial, we proved in [8] that $R_{n}\left(\theta_{r}(h)\right)=O(1 / \log n)$. Combining this with our result in the present paper, we have $R_{n}\left(\theta_{r}(h)\right)=O(1 / \log n)$ for all functions $h(t)$ given above; in particular, the number $\theta_{r}(h)$ is normal to the base $r$ for all $h(t)$.

Remark 2. Our method of the proof in [9], which is quite different from that of Schiffer [10], made use of an estimate of Weyl sums in a somewhat unusual manner and of simple remarks on diophantine approximation. In this paper, we further develop this method by employing inductive arguments and we obtain the improved results. As for the proof of the result in [8], tricky estimates for exponential sums of Vinogradov type were used.

\section{Lemmas}

Lemma 1 ([9], Corollary of Lemma). Let $p(t)$ be a polynomial with real coefficients and the leading term $\gamma t^{k}$, where $\gamma \neq 0$ and $k \geq 1$. Let $Q \geq 2$ and let $A / B$ be a rational number with $(A, B)=1$ such that

$$
(\log Q)^{h} \ll B \ll Q^{k}(\log Q)^{-h},
$$


and

$$
|\gamma-A / B| \leq B^{-2},
$$

where $h \geq(k-1)^{2}+2^{k} G$ with $G>0$. Then

$$
\left|\sum_{1 \leq n \leq Q} e(p(n))\right| \ll Q(\log Q)^{-G},
$$

where $e(t)=e^{2 \pi i t}$.

LEMMA 2. Let $f(t)$ be a polynomial of the form

$$
f(t)=\beta_{0} t^{k_{0}}+\beta_{1} t^{k_{1}}+\ldots+\beta_{d} t^{k_{d}},
$$

where $k_{0}>k_{1}>\ldots>k_{d} \geq 1$ and $\beta_{0}, \ldots, \beta_{d}$ are nonzero real numbers. Let $G>0$ be any constant and $X \geq 2$. Let $s$ be an integer with $0 \leq s \leq d$, let $H_{i}, K_{i}(i=0,1, \ldots, s-1)$ be any positive constants, and let $H_{s}^{*}, K_{s}^{*}$ be constants such that

$$
\begin{aligned}
& H_{s}^{*} \geq 2^{k_{s}+1}\left(G+\max _{0 \leq i<s} H_{i}+1\right)+k_{s} \sum_{i=0}^{s-1} K_{i}, \\
& K_{s}^{*} \geq 2^{k_{s}+1}\left(G+\max _{0 \leq i<s} H_{i}+1\right)+2 k_{s} \sum_{i=0}^{s-1} K_{i} .
\end{aligned}
$$

Suppose that there are rational numbers $A_{i} / B_{i}(0 \leq i<s)$ such that

$$
1 \leq B_{i} \leq(\log X)^{K_{i}} \quad \text { and } \quad\left|\beta_{i}-\frac{A_{i}}{B_{i}}\right| \leq \frac{(\log X)^{H_{i}}}{B_{i} X^{k_{i}}} \quad(0 \leq i<s)
$$

and that there is no rational number $A_{s} / B_{s}$ with $\left(A_{s}, B_{s}\right)=1$ such that

$$
1 \leq B_{s} \leq(\log X)^{K_{s}^{*}} \quad \text { and } \quad\left|\beta_{s}-\frac{A_{s}}{B_{s}}\right| \leq \frac{(\log X)^{H_{s}^{*}}}{B_{s} X^{k_{s}}} .
$$

Then, for any real $P$ and $Q$ with $|P| \ll Q \leq X$,

$$
\left|\sum_{P<n \leq P+Q} e(f(n))\right| \ll X(\log X)^{-G} .
$$

Proof. We may assume $P=0$ and

$$
X(\log X)^{-G} \leq Q \leq X .
$$

If $s=0$, the inequality follows immediately from Lemma 1 . We put $p(t)=$ $f(t)$, so that $\gamma=\beta_{0}$ and $k=k_{0}$. Since $s=0, \max _{0 \leq i<s} H_{i}=\sum_{i=0}^{s-1} K_{i}=0$. We choose, by the well-known argument, a rational number $A / B$ with $(A, B)=1$ such that

$$
1 \leq B \leq \frac{X^{k}}{(\log X)^{H_{0}^{*}}} \quad \text { and } \quad\left|\gamma-\frac{A}{B}\right| \leq \frac{(\log X)^{H_{0}^{*}}}{B X^{k}} \quad\left(\leq B^{-2}\right),
$$


where $H_{0}^{*}, K_{0}^{*} \geq 2^{k+1}(G+1)$. Then by the assumption, we have $B \geq$ $(\log X)^{K_{0}^{*}}$. These inequalities as well as (6) imply (5) with $h=(k-1)^{2}+2^{k} G$. Therefore we obtain

$$
\left|\sum_{1 \leq n \leq Q} e(f(n))\right| \ll Q(\log Q)^{-G} \ll X(\log X)^{-G} .
$$

Let $s \geq 1$. We denote by $D$ the least common multiple of $B_{0}, \ldots, B_{s-1}$ and by $N$ the integer defined by $D N \leq Q<D(N+1)$, so that

$$
1 \leq D \leq(\log X)^{K} \quad \text { with } K=\sum_{i=0}^{s-1} K_{i}
$$

and by (6)

$$
X(\log X)^{-(G+K)} \ll N \gg \ll Q / D \leq X / D .
$$

It follows that

$$
\sum_{1 \leq n \leq Q} e(f(n))=\sum_{\lambda=0}^{D-1} \sum_{\nu=1}^{N} e(f(\lambda+D \nu))+O\left((\log X)^{K}\right) .
$$

We put

$$
\begin{gathered}
f_{\lambda}(y)=\sum_{i=0}^{s-1} \Omega_{i}(\lambda+D y)^{k_{i}}, \quad \Omega_{i}=\beta_{i}-A_{i} / B_{i}, \\
\varphi_{\lambda}(y)=\sum_{i=s}^{d} \beta_{i}(\lambda+D y)^{k_{i}}, \quad T_{\lambda}(\nu)=\sum_{n=1}^{\nu} e\left(\varphi_{\lambda}(n)\right) .
\end{gathered}
$$

Then we have

$$
\begin{aligned}
\sum_{\lambda=0}^{D-1} \sum_{\nu=1}^{N} e(f(\lambda+ & D \nu)) \\
= & \sum_{\lambda=0}^{D-1} e\left(\sum_{i=0}^{s-1} \frac{A_{i}}{B_{i}} \lambda^{k_{i}}\right) \sum_{\nu=1}^{N} e\left(f_{\lambda}(\nu)\right)\left(T_{\lambda}(\nu)-T_{\lambda}(\nu-1)\right) \\
= & \sum_{\lambda=0}^{D-1} e\left(\sum_{i=0}^{s-1} \frac{A_{i}}{B_{i}} \lambda^{k_{i}}\right)\left\{e\left(f_{\lambda}(N+1)\right) T_{\lambda}(N)\right. \\
& \left.+\sum_{\nu=1}^{N}\left(e\left(f_{\lambda}(\nu)\right)-e\left(f_{\lambda}(\nu+1)\right)\right) T_{\lambda}(\nu)\right\} \\
\ll & \sum_{\lambda=0}^{D-1}\left(\left|T_{\lambda}(N)\right|+\sum_{\nu=1}^{N}\left|e\left(f_{\lambda}(\nu)\right)-e\left(f_{\lambda}(\nu+1)\right)\right|\left|T_{\lambda}(\nu)\right|\right) .
\end{aligned}
$$


Here we have, using the mean-value theorem,

$$
\left|e\left(f_{\lambda}(\nu)\right)-e\left(f_{\lambda}(\nu+1)\right)\right| \ll D \sum_{i=0}^{s-1}\left|\Omega_{i}\right| Q^{k_{i}-1} \ll D \frac{(\log X)^{H}}{X}
$$

with

Therefore we obtain

$$
H=\max _{0 \leq i<s} H_{i}
$$

(8) $\sum_{\lambda=0}^{D-1} \sum_{\nu=1}^{N} e(f(\lambda+D \nu)) \ll \sum_{\lambda=0}^{D-1}\left(\left|T_{\lambda}(N)\right|+D \frac{(\log X)^{H}}{X} \sum_{\nu=1}^{N}\left|T_{\lambda}(\nu)\right|\right)$.

We next prove that

$$
\left|T_{\lambda}(\nu)\right|=\left|\sum_{n=1}^{\nu} e\left(\varphi_{\lambda}(n)\right)\right| \ll \frac{X}{D(\log X)^{G+H}}
$$

for all $\nu$ with $1 \leq \nu \leq N$. For this, we may assume that

$$
\frac{X}{D(\log X)^{G+H}} \ll \nu \quad(\leq N \leq X / D) .
$$

We put $p(t)=\varphi_{\lambda}(t)$ in Lemma 1 , so that the leading coefficient is $\gamma=$ $D^{k_{s}} \beta_{s}$. Suppose first that there is a rational number $A / B$ with $(A, B)=1$ such that

$$
(\log X)^{H^{\prime}} \leq B \leq X^{k_{s}}(\log X)^{-H^{\prime}}
$$

and

$$
|\gamma-A / B| \leq B^{-2}
$$

where $H^{\prime}=2^{k_{s}+1}(G+H+1)+k_{s} K$. Then (11) together with (10) implies

$$
(\log \nu)^{h^{\prime}} \leq B \leq \nu^{k_{s}}(\log \nu)^{-h^{\prime}},
$$

where $h^{\prime}=\left(k_{s}-1\right)^{2}+2^{k_{s}}(G+H)$. Hence we have by Lemma 1

$$
\left|T_{\lambda}(\nu)\right| \ll \nu(\log \nu)^{-(G+H)} \ll \frac{X}{D(\log X)^{G+H}} .
$$

If there is no such rational number, we can choose a rational number $A^{\prime} / B^{\prime}$ with $\left(A^{\prime}, B^{\prime}\right)=1$ such that

$$
1 \leq B^{\prime} \leq(\log X)^{H^{\prime}} \quad \text { and } \quad\left|\gamma-\frac{A^{\prime}}{B^{\prime}}\right| \leq \frac{(\log X)^{H^{\prime}}}{B^{\prime} X^{k_{s}}} .
$$

Then we have

$$
D^{k_{s}} B^{\prime} \leq(\log X)^{H^{\prime}+k_{s} K} \leq(\log X)^{K_{s}^{*}}
$$


and

which contradicts the assumption on $\beta_{s}$.

$$
\left|\beta_{s}-\frac{A^{\prime}}{D^{k_{s}} B^{\prime}}\right| \leq \frac{(\log X)^{H_{s}^{*}}}{D^{k_{s}} B^{\prime} X^{k_{s}}},
$$

Combining (7), (8), and (9), we obtain

$$
\begin{aligned}
\left|\sum_{1 \leq n \leq Q} e(f(n))\right| & \ll(\log X)^{H}+\sum_{\lambda=0}^{D-1}\left(1+D N \frac{(\log X)^{H}}{X}\right) \frac{X}{D(\log X)^{G+H}} \\
& \ll X(\log X)^{-G},
\end{aligned}
$$

and the proof is complete.

3. Preliminaries of the proof of theorem. Let $g(t)$ be as in the theorem. Let $j_{0}$ be an integer chosen sufficiently large. Then, for each $j \geq j_{0}$, there is a positive integer $n_{j}$ such that $r^{j-2} \leq g\left(n_{j}\right)<r^{j-1} \leq g\left(n_{j}+1\right)<r^{j}$. It follows that $n_{j}<n \leq n_{j+1}$ if and only if $r^{j-1} \leq g(n)<r^{j}$ and that

$$
n_{j} \gg \ll r^{j / k}, \quad n_{j+1}-n_{j} \gg \ll r^{j / k},
$$

where $k \geq 1$ is the degree of the polynomial $g(t)$. Let $x>r^{j_{0}}$ and let $J$ be a positive integer such that $n_{J}<x \leq n_{J+1}$, so that

$$
J=\log _{r} g(x)+O(1)=O(\log x) .
$$

Put $X_{J}=x-n_{J}$ and $X_{j}=n_{j+1}-n_{j}$ for $\left(j_{0} \leq\right) j \leq J-1$. We write $N(g(n))=N\left(g(n) ; b_{1} \ldots b_{l}\right)$. Then

$$
\sum_{n \leq x} N(g(n))=\sum_{j_{0} \leq j \leq J} \sum_{n_{j}<n \leq n_{j}+X_{j}} N(g(n))+O(1) .
$$

Defining the periodic function $I(t)$ with period 1 by

$$
I(t)= \begin{cases}1 & \text { if } \sum_{h=1}^{l} \frac{b_{h}}{r^{h}} \leq t-[t]<\sum_{h=1}^{l} \frac{b_{h}}{r^{h}}+\frac{1}{r^{l}}, \\ 0 & \text { otherwise }\end{cases}
$$

we have

$$
\sum_{n_{j}<n \leq n_{j}+X_{j}} N(g(n))=\sum_{l \leq m \leq j} \sum_{n_{j}<n \leq n_{j}+X_{j}} I\left(\frac{g(n)}{r^{m}}\right) .
$$

Let $j$ be any integer with $j_{0} \leq j \leq J$ and let $C$ be a constant chosen sufficiently large.

In this section, we treat those $m$ with $C \log j \leq m \leq j-C \log j$. There are, for each $j$, functions $I_{-}(t)$ and $I_{+}(t)$, periodic with period 1 , such that 
$I_{-}(t) \leq I(t) \leq I_{+}(t)$, having Fourier expansion of the form

$$
I_{ \pm}(t)=r^{-l} \pm j^{-1}+\sum_{\substack{\nu=-\infty \\ \nu \neq 0}}^{\infty} A_{ \pm}(\nu) e(\nu t)
$$

with $\left|A_{ \pm}(\nu)\right| \ll \min \left(|\nu|^{-1}, j|\nu|^{-2}\right)$ (cf. [14]).

We shall estimate the exponential sums

$$
S(j, m, \nu)=\sum_{n=n_{j}+1}^{n_{j}+X_{j}} e\left(\frac{\nu}{r^{m}} g(n)\right),
$$

where $J \geq j \geq j_{0}, j-C \log j \geq m \geq C \log j$, and $1 \leq \nu \leq j^{2}$. Here the leading coefficient of $\nu r^{-m} g(t)$ is $\nu r^{-m} \alpha$. Assume first that $j<J$. For any pair $(m, \nu)$ for which there is a rational number $a / q$ such that

$$
\begin{aligned}
& (a, q)=1, \quad\left|\frac{\nu}{r^{m}} \alpha-\frac{a}{q}\right| \leq \frac{1}{q^{2}}, \\
& \left(\log X_{j}\right)^{H} \leq q \leq X_{j}^{b}\left(\log X_{j}\right)^{-H}
\end{aligned}
$$

with $G=3$ and $H$ as in Lemma 1 , we have

$$
|S(j, m, \nu)| \ll X_{j}\left(\log X_{j}\right)^{-3} \ll X_{j} j^{-3}
$$

by Lemma 1 . Hence, denoting by $\sum^{\prime}$ the sum over all pairs $(m, \nu)$ having this property, we have the following estimates:

$$
\sum_{m} \sum_{\nu}{ }^{\prime} \min \left(\nu^{-1}, J \nu^{-2}\right)|S(j, m, \nu)| \ll j \log j \cdot X_{j} j^{-3} \ll X_{j} \ll r^{j / b} .
$$

If $j=J$, there are two cases. Assume first that $X_{J}=O\left(r^{J / b} J^{-3}\right)$. Then we have trivial estimates

$$
\sum_{m=l}^{J} \sum_{\nu=1}^{J^{2}} \min \left(\nu^{-1}, J \nu^{-2}\right)|S(J, m, \nu)| \ll r^{J / b} J^{-1} .
$$

Otherwise, namely if $X_{J} \gg r^{J / b} J^{-3}$, then $\log X_{J} \gg \ll J$, so that we can repeat the same argument as for $j<J$. In any case, we get

$$
\sum_{m} \sum_{\nu}{ }^{\prime} \min \left(\nu^{-1}, j \nu^{-2}\right)|S(j, m, \nu)| \ll r^{j / b}
$$

for $\left(j_{0} \leq\right) j \leq J$ (see $[9 ;$ p. 208]).

On the other hand, if $(j \geq) m \geq(j / \beta)(\beta-\delta)$ with a small positive constant $\delta$, we can appeal to Lemmas 4.2 and 4.8 of [12], with $f(t)=$ $\nu r^{-m} g(t)$. Then, for these $m$ and $\nu \leq j^{2}$, we have, with positive constants $c_{0}$ and $c_{1}$,

$$
0<c_{0} \nu r^{-m+j(1-1 / \beta)}<f^{\prime}(t)<c_{1} \nu r^{-m+j(1-1 / \beta)}<1 / 2
$$


throughout the interval $\left[n_{j}, n_{j}+X_{j}\right]$, since

$$
j\left(1-\frac{1}{\beta}\right)-m \leq j\left(1-\frac{1}{\beta}\right)-j\left(1-\frac{\delta}{\beta}\right)<\frac{\delta-1}{\beta}<0 .
$$

Hence by the lemmas cited,

$$
|S(j, m, \nu)|=O\left(\frac{1}{\nu} r^{j / \beta+m-j}\right)
$$

provided $(j / \beta)(\beta-\delta) \leq m \leq j$ and $1 \leq \nu \leq j^{2}$ (see [8; p. 26]).

Thus it is proved that

$$
\sum_{C \log j \leq m \leq j} \sum_{n_{j}<n \leq n_{j}+X_{j}}\left(I\left(\frac{g(n)}{r^{m}}\right)-\frac{1}{r^{l}}\right)=O\left(r^{j / k}\right) .
$$

Therefore, if we can prove the inequality

$$
\sum_{l \leq m \leq C \log j} \sum_{n_{j}<n \leq n_{j}+X_{j}}\left(I\left(\frac{g(n)}{r^{m}}\right)-\frac{1}{r^{l}}\right)=O\left(r^{j / k}\right),
$$

we shall have obtained

$$
\sum_{l \leq m \leq j} \sum_{n_{j}<n \leq n_{j}+X_{j}} I\left(\frac{g(n)}{r^{m}}\right)=\frac{1}{r^{l}} j X_{j}+O\left(r^{j / k}\right),
$$

which leads to

$$
\sum_{n \leq x} N(g(n))=\frac{1}{r^{l}} x J+O\left(r^{J / k}\right)=\frac{1}{r^{l}} x \log _{r} g(x)+O(x),
$$

which is the assertion of our theorem. Thus it remains to show (14).

4. Proof of the inequality (14). In this section, we shall prove (14) for those $j$ for which at least one of the coefficients of $g(t)$ has no rational approximations with small denominators in the sense stated in Lemma 2.

To estimate the sum

$$
\sum_{n_{j}<n \leq n_{j}+X_{j}} I\left(\frac{g(n)}{r^{m}}\right)
$$

in (14), we approximate the function $I(t)$ by functions $I_{-}(t)$ and $I_{+}(t)$ periodic with period 1 , such that $I_{-}(t) \leq I(t) \leq I_{+}(t)$, having Fourier expansion of the form

$$
I_{ \pm}(t)=\frac{1}{r^{l}} \pm \frac{1}{j}+\sum_{\nu \in \mathbb{Z}, \nu \neq 0} A_{ \pm}(\nu) e(\nu t)
$$

with $\left|A_{ \pm}(\nu)\right| \ll \min \left(|\nu|^{-1}, j \nu^{-2}\right)$, where the constant implied is absolute 
(cf. [14]). Then we have

$$
\begin{aligned}
& \sum_{n_{j}<n \leq n_{j}+X_{j}} I\left(\frac{g(n)}{r^{m}}\right) \\
& \left.=\frac{X_{j}}{r^{l}}+O\left(\frac{X_{j}}{j}\right)+O\left(\sum_{\nu=1}^{j^{2}} \frac{1}{\nu} \mid \sum_{n_{j}<n \leq n_{j}+X_{j}} e\left(\frac{\nu}{r^{m}} g(n)\right)\right)\right) .
\end{aligned}
$$

We shall evaluate

$$
\left|\sum_{n_{j}<n \leq n_{j}+X_{j}} e\left(\frac{\nu}{r^{m}} g(n)\right)\right|
$$

with $l \leq m \leq C \log j$ and $1 \leq \nu \leq j^{2}$, by making use of Lemma 2 inductively.

Let the polynomial $g(t)$ be of the form

$$
g(t)=\alpha_{0} t^{k_{0}}+\alpha_{1} t^{k_{1}}+\ldots+\alpha_{d} t^{k_{d}}
$$

where $k=k_{0}>k_{1}>\ldots>k_{d} \geq 0$ and $\alpha_{0}, \ldots, \alpha_{d}$ are nonzero real numbers. We may assume $k_{d} \geq 1$ in estimating the exponential sum above. We put in Lemma 2

$$
f(t)=r^{-m} \nu g(t)
$$

so that

$$
\beta_{i}=r^{-m} \nu \alpha_{i} \quad(0 \leq i \leq d) .
$$

We choose a constant $c>0$ such that $c r^{j / k} \geq X_{j}$ for all $j \leq J$, and define a parameter $X$ by

$$
X=X(j)=c r^{j / k} \quad\left(j_{0} \leq j \leq J\right) .
$$

Then $\log X=j^{1+o(1)}$, as $j \rightarrow \infty$, so that

$$
r^{m} \leq(\log X)^{C \log r+o(1)}, \quad \nu \leq(\log X)^{2+o(1)},
$$

since $m \leq C \log j$ and $\nu \leq j^{2}$.

Case 0 . Let $j$ be an integer with $j_{0} \leq j \leq J$ for which there is no rational number $a_{0} / b_{0}$ with $\left(a_{0}, b_{0}\right)=1$ such that

$$
1 \leq b_{0} \leq(\log X)^{2 h_{0}} \quad \text { and } \quad\left|\alpha_{0}-\frac{a_{0}}{b_{0}}\right| \leq \frac{(\log X)^{h_{0}}}{b_{0} X^{k_{0}}},
$$

where

$$
h_{0}=H_{0}^{*}+C \log r+1, \quad H_{0}^{*}=2^{k_{0}+1}(G+1) .
$$

The set of all $j$ with this property will be denoted by $\mathbb{J}_{0}$. If $j \in \mathbb{J}_{0}$, there is no rational number $A_{0} / B_{0}$ with $\left(A_{0}, B_{0}\right)=1$ such that

$$
1 \leq B_{0} \leq(\log X)^{2 H_{0}^{*}} \quad \text { and } \quad\left|\beta_{0}-\frac{A_{0}}{B_{0}}\right| \leq \frac{(\log X)^{H_{0}^{*}}}{B_{0} X^{k_{0}}}
$$


since, if there is such a rational number $A_{0} / B_{0}$, we shall have

$$
1 \leq \nu B_{0} \leq(\log X)^{2 H_{0}^{*}+3} \leq(\log X)^{2 h_{0}}
$$

and

$$
\left|\alpha_{0}-\frac{r^{m} A_{0}}{\nu B_{0}}\right| \leq \frac{(\log X)^{H_{0}^{*}+C \log r+1}}{\nu B_{0} X^{k_{0}}} \leq \frac{(\log X)^{h_{0}}}{\nu B_{0} X^{k_{0}}},
$$

which contradicts the assumptions in this case. Hence we can apply Lemma 2 with $s=0$ and obtain

$$
\left|\sum_{n_{j}<n \leq n_{j}+X_{j}} e\left(\frac{\nu}{r^{m}} g(n)\right)\right| \ll \frac{X}{(\log X)^{G}}
$$

for all $j \in \mathbb{J}_{0}$.

Case $s$. Let $1 \leq s \leq d$. We put

$$
H_{0}^{*}=2^{k_{0}+1}(G+1), \quad H_{0}=H_{0}^{*}+2^{k_{0}+1}(G+1)
$$

and define $H_{i}^{*}$ and $H_{i}(1 \leq i \leq d)$ inductively by

$$
\begin{aligned}
H_{i}^{*} & =2^{k_{i}+1}\left(G+H_{i-1}+1\right)+2 k_{i}\left(H_{0}+\ldots+H_{i-1}\right), \\
H_{i} & =H_{i}^{*}+2(C \log r+1) .
\end{aligned}
$$

Also we write

$$
h_{i}=H_{i}^{*}+C \log r+1 \quad(0 \leq i \leq d) .
$$

Let $j$ be an integer with $j_{0} \leq j \leq J$ for which there are rational numbers $a_{0} / b_{0}, \ldots, a_{s-1} / b_{s-1}$ such that

$$
1 \leq b_{i} \leq(\log X)^{2 h_{i}} \quad \text { and } \quad\left|\alpha_{i}-\frac{a_{i}}{b_{i}}\right| \leq \frac{(\log X)^{h_{i}}}{b_{i} X^{k_{i}}} \quad(0 \leq i<s)
$$

but there is no rational number $a_{s} / b_{s}$ with $\left(a_{s}, b_{s}\right)=1$ such that

$$
1 \leq b_{s} \leq(\log X)^{2 h_{s}} \quad \text { and } \quad\left|\alpha_{s}-\frac{a_{s}}{b_{s}}\right| \leq \frac{(\log X)^{h_{s}}}{b_{s} X^{k_{s}}} .
$$

The set of all $j$ with this property will be denoted by $\mathbb{J}_{s}$. If $j \in \mathbb{J}_{s}$, we have

$$
1 \leq r^{m} b_{i} \leq(\log X)^{2 H_{i}} \quad \text { and } \quad\left|\beta_{i}-\frac{\nu a_{i}}{r^{m} b_{i}}\right| \leq \frac{(\log X)^{H_{i}}}{r^{m} b_{i} X^{k_{i}}}
$$

for $0 \leq i<s$, but there is no rational number $A_{s} / B_{s}$ with $\left(A_{s}, B_{s}\right)=1$ such that

$$
1 \leq B_{s} \leq(\log X)^{2 H_{s}^{*}} \quad \text { and } \quad\left|\beta_{s}-\frac{A_{s}}{B_{s}}\right| \leq \frac{(\log X)^{H_{s}^{*}}}{B_{s} X^{k_{s}}},
$$

since otherwise we have a contradiction as in Case 0. Hence, by Lemma 2 with these $H_{i}, H_{s}^{*}$ and $K_{i}=2 H_{i}, K_{s}^{*}=2 H_{s}^{*}$, we have again (15) for all $j \in \mathbb{J}_{s}$. 
Choosing $G=3$ in (15), we get

$$
\left|\sum_{n_{j}<n \leq n_{j}+X_{j}} e\left(\frac{\nu}{r^{m}} g(n)\right)\right| \ll \frac{r^{j / k}}{j^{2}}
$$

for all $(l \leq) m \leq C \log j,(1 \leq) \nu \leq j^{2}$, and $j \in \mathbb{J}_{0} \cup \ldots \cup \mathbb{J}_{d}$, and hence by (14)

$$
\sum_{l \leq m \leq C \log j} \sum_{n_{j}<n \leq n_{j}+X_{j}}\left(I\left(\frac{g(n)}{r^{m}}\right)-\frac{1}{r^{l}}\right)=O\left(\frac{r^{j / k}}{j}\right)
$$

for all $j \in \mathbb{J}_{0} \cup \ldots \cup \mathbb{J}_{d}$.

It remains to prove (14) for $j \notin \mathbb{J}_{0} \cup \ldots \cup \mathbb{J}_{d}$ with $j_{0} \leq j \leq J$, which will be done in the next section.

5. Proof of the inequality (14). Continued. Let $\mathbb{J}_{d+1}$ be the set of all integers $j$ with $j_{0} \leq j \leq J$ for which there are rational numbers $a_{i} / b_{i}$ with $\left(a_{i}, b_{i}\right)=1$ such that

$$
1 \leq b_{i} \leq(\log X)^{2 h_{d}} \quad \text { and } \quad\left|\alpha_{i}-\frac{a_{i}}{b_{i}}\right| \leq \frac{(\log X)^{h_{d}}}{b_{i} X^{k_{i}}}
$$

for all $i=0,1, \ldots, d$, where $h_{d}$ is defined in Section 4 . Then by definition

$$
\left\{j_{0}, j_{0}+1, \ldots, J\right\}=\mathbb{J}_{0} \cup \ldots \cup \mathbb{J}_{d} \cup \mathbb{J}_{d+1} .
$$

In the rest of this paper, we shall prove (14) for all $j \in \mathbb{J}_{d+1}$ by a method different from that used in the preceding section. We assume $k_{d} \geq 1$. The proof is valid also in the case of $k_{d}=0$.

Let $j \in \mathbb{J}_{d+1}$. We denote by $a_{*}$ the greatest common divisor of $a_{0}, \ldots, a_{d}$ and by $b^{*}$ the least common multiple of $b_{0}, \ldots, b_{d}$. Then $\left(a_{*}, b^{*}\right)=1$ and

$$
1 \leq b^{*} \leq j^{h}, \quad 1 \leq a_{*} \ll j^{h},
$$

where $h=2(d+1) h_{d}+1$. We then define integers $c_{0}, \ldots, c_{d}$ by

$$
\frac{a_{i}}{b_{i}}=\frac{a_{*} c_{i}}{b^{*}}
$$

so that $\left(b^{*}, a_{*} c_{0}, \ldots, a_{*} c_{d}\right)=1$. We write for brevity $L_{1}=\log j$ and $L_{w}=$ $\log L_{w-1}\left(2 \leq w \leq w_{j}\right)$, where $w_{j}$ is the greatest integer $w$ for which $L_{w} \geq 3$.

For a given positive constant $C$, we have

$$
\begin{aligned}
& \sum_{l \leq m \leq C \log j}\left|\sum_{n_{j}<n \leq n_{j}+X_{j}}\left(I\left(r^{-m} g(n)\right)-r^{-l}\right)\right| \\
\leq & \sum_{1 \leq w \leq w_{j}} \sum_{V L_{w+1}<m \leq V L_{w}}\left|\sum_{n_{j}<n \leq n_{j}+X_{j}}\left(I\left(r^{-m} g(n)\right)-r^{-l}\right)\right|+V X_{j}
\end{aligned}
$$

where $V \geq C$ is a constant which will be chosen suitably at the end of the proof. For each $w$, there are functions $I_{w}^{-}(t)$ and $I_{w}^{+}(t)$, periodic with 
period 1, such that $I_{w}^{-}(t) \leq I(t) \leq I_{w}^{+}(t)$, having Fourier expansion of the form

$$
I_{w}^{ \pm}(t)=r^{-l} \pm L_{w}^{-2}+\sum_{\nu \in \mathbb{Z}, \nu \neq 0} A_{w}^{ \pm}(\nu) e(\nu t)
$$

with $\left|A_{w}^{ \pm}(\nu)\right| \leq \min \left(|\nu|^{-1}, L_{w}^{2} \nu^{-2}\right)$ (cf. [14]). Then it follows that

$$
\begin{aligned}
\sum_{n_{j}<n \leq n_{j}+X_{j}}\left(I\left(r^{-m} g(n)\right)-r^{-l}\right) & \\
& \ll X_{j} L_{w}^{-2}+\sum_{1 \leq \nu \leq L_{w}^{4}} \nu^{-1}\left|\sum_{n_{j}<n \leq n_{j}+X_{j}} e\left(r^{-m} \nu g(n)\right)\right| .
\end{aligned}
$$

Here we have, for any fixed $m$ with $V L_{w+1}<m \leq V L_{w}$ and $\nu$ with $1 \leq \nu \leq L_{w}^{4}$

$$
\begin{aligned}
& \sum_{n_{j}<n \leq n_{j}+X_{j}} e\left(r^{-m} \nu g(n)\right) \\
= & \sum_{0 \leq \lambda \leq r^{m} b^{*}} e\left(\frac{\nu a_{*}}{r^{m} b^{*}} \sum_{i=0}^{d} c_{i} \lambda^{k_{i}}\right) \sum_{\substack{\nu ; n=\lambda+r^{m} b^{*} \nu \\
n_{j}<n \leq n_{j}+X_{j}}} e\left(\frac{\nu}{r^{m}} \sum_{i=0}^{d} \Omega_{i} n^{k_{i}}\right) \\
= & \sum_{0 \leq \lambda \leq r^{m} b^{*}} e\left(\frac{\nu a_{*}}{r^{m} b^{*}} \sum_{i=0}^{d} c_{i} \lambda^{k_{i}}\right)\left\{\int_{\substack{n_{j}<n \leq n_{j}+X_{j} \\
x=\lambda+r^{m} b^{*} y}} e\left(\frac{\nu}{r^{m}} \sum_{i=0}^{d} \Omega_{i} x^{k_{i}}\right) d y+O(1)\right\} \\
= & \sum_{0 \leq \lambda \leq r^{m} b^{*}} e\left(\frac{\nu a_{*}}{r^{m} b^{*}} \sum_{i=0}^{d} c_{i} \lambda^{k_{i}}\right) \frac{1}{r^{m} b^{*}} \int_{\substack{n_{j}<n \leq n_{j}+X_{j} \\
n^{m}}} e\left(\frac{\nu}{i=0} \sum_{i} x^{k_{i}}\right) d x \\
& +O\left(r^{m} b^{*}\right),
\end{aligned}
$$

using a lemma of van der Corput's ([12], Lemma 4.8), where $\Omega_{i}=\alpha_{i}-a_{i} / b_{i}$. Defining now rational numbers $R_{i} / Q(0 \leq i \leq d)$ by

$$
\frac{R_{i}}{Q}=\frac{\nu}{r^{m}} \frac{a_{*} c_{i}}{b^{*}}\left(=\frac{\nu}{r^{m}} \frac{a_{i}}{b_{i}}\right) \quad \text { with }\left(Q, R_{0}, R_{1}, \ldots, R_{d}\right)=1
$$

and applying the theorem in [4], Chap. $1, \S 1$, to the exponential sum over $\lambda$, we get

$$
\begin{aligned}
\sum_{n_{j}<n \leq n_{j}+X_{j}} e\left(r^{-m} \nu g(n)\right) & \ll \frac{r^{m} b^{*}}{Q} Q^{1-9 /(10 k)} \frac{X_{j}}{r^{m} b^{*}}+r^{m} b^{*} \\
& \ll X_{j} Q^{-9 /(10 k)}+r^{m} j^{h}
\end{aligned}
$$


and hence by (17)

(18)

$$
\begin{aligned}
& \sum_{V L_{w+1}<m \leq V L_{w}}\left|\sum_{n_{j}<n \leq n_{j}+X_{j}}\left(I\left(r^{-m} g(n)\right)-r^{-l}\right)\right| \\
& \ll \sum_{V L_{w+1}<m \leq V L_{w}}\left(X_{j} L_{w}^{-2}+X_{j} \sum_{1 \leq \nu \leq L_{w}^{4}} \nu^{-1} Q^{-9 /(10 k)}+L_{w+1} r^{m} j^{h}\right) \\
& \ll r^{j / k} L_{w}^{-1}+X_{j} \sum_{V L_{w+1}<m \leq V L_{w}} \sum_{1 \leq \nu \leq L_{w}^{4}} \nu^{-1} Q^{-9 /(10 k)} .
\end{aligned}
$$

Therefore it follows from (16) and (18) that

$$
\begin{aligned}
& \sum_{l \leq m \leq C \log j}\left|\sum_{n_{j}<n \leq n_{j}+X_{j}}\left(I\left(r^{-m} g(n)\right)-r^{-l}\right)\right| \\
& \ll r^{j / k}+r^{j / k} \sum_{1 \leq w \leq w_{j} V L_{w+1}<m \leq V L_{w}} \sum_{1 \leq \nu \leq L_{w}^{4}} \nu^{-1} Q^{-9 /(10 k)} .
\end{aligned}
$$

But, since $\nu Q=r^{m} R_{i} b_{i} / a_{i} \gg \ll r^{m} R_{i} \alpha_{i}^{-1} \gg \ll r^{m} R_{i} \gg r^{m}$ by the definition of $R_{i} / Q$, we obtain

$$
\begin{aligned}
\sum_{1 \leq w \leq w_{j}} \sum_{V L_{w+1}<m \leq V L_{w}} & \sum_{1 \leq \nu \leq L_{w}^{4}} \nu^{-1} Q^{-9 /(10 k)} \\
& \ll \sum_{1 \leq w \leq w_{j}} \sum_{V L_{w+1}<m \leq V L_{w}} \sum_{1 \leq \nu \leq L_{w}^{4}}\left(r^{m}\right)^{-9 /(10 k)} \\
& \ll \sum_{1 \leq w \leq w_{j}} V L_{w} \cdot L_{w}^{4}\left(r^{V L_{w+1}}\right)^{-9 /(10 k)} \\
& \ll V \sum_{1 \leq w \leq w_{j}} L_{w}^{5-\frac{9 \log r}{10 k} V} \ll V \sum_{1 \leq w \leq w_{j}} L_{w}^{-1} \ll 1,
\end{aligned}
$$

provided that $V \geq \max (C, 20 k /(3 \log r))$. Combining this with (19), we have (14) for all $j \in \mathbb{J}_{d+1}$. Therefore, (14) is proved for any $j$ with $j_{0} \leq j \leq J$, and the proof of the theorem is complete.

\section{References}

[1] A. S. Besicovitch, The asymptotic distribution of the numerals in the decimal representation of the squares of the natural numbers, Math. Z. 39 (1935), 146-156.

[2] D. G. Champernowne, The construction of decimals normal in the scale of ten, J. London Math. Soc. 8 (1933), 254-260.

[3] H. Daven port and P. Erdős, Note on normal decimals, Canad. J. Math. 4 (1952), $58-63$.

[4] L.-K. Hua, Additive Theory of Prime Numbers, Transl. Math. Monographs 13, Amer. Math. Soc., Providence, Rhode Island, 1965. 
[5] A. A. Karatsuba, Foundations of Analytic Theory of Numbers, 2nd ed., Nauka, 1983 (in Russian).

[6] L. Kuipers and H. Niederreiter, Uniform Distribution of Sequences, Wiley, New York 1974.

[7] L. Mirsky, A theorem on representations of integers in the scale of $r$, Scripta Math. 15 (1974), 11-12.

[8] Y.-N. Nakai and I. Shiokawa, A class of normal numbers, Japan. J. Math. 16 (1990), 17-29.

[9] _- - A class of normal numbers II, in: Number Theory and Cryptography, J. H. Loxton (ed.), London Math. Soc. Lecture Note Ser. 154, Cambridge Univ. Press, 1990, 204-210.

[10] J. Schiffer, Discrepancy of normal numbers, Acta Arith. 47 (1986), 175-186.

[11] J. Schoißengeier, Über die Diskrepanz von Folgen $\left(\alpha b^{n}\right)$, Sitzungsber. Öst. Akad. Wiss. Math.-Natur. Kl. Abt. II 187 (1978), 225-235.

[12] E. C. Titchmarsh, The Theory of the Riemann Zeta-Function, Oxford Univ. Press, 1951.

[13] R. C. Vaughan, The Hardy-Littlewood Method, Cambridge Tracts in Math. 80, Cambridge Univ. Press, London 1981.

[14] I. M. Vinogradov, The Method of Trigonometric Sums in Number Theory, Nauka, 1971 (in Russian).

DEPARTMENT OF MATHEMATICS

DEPARTMENT OF MATHEMATICS

FACULTY OF EDUCATION

KEIO UNIVERSITY

YAMANASHI UNIVERSITY

HIYOSHI, YOKOHAMA 223

KOFU, 400 JAPAN

JAPAN

Received on 25.7.1991

and in revised form on 3.1 .1992 